\title{
Comparative Study of Antenna Elements for TDRSS Enhanced Multiple Access System
}

\author{
Carol L. Kory*(1), Kevin Lambert ${ }^{(1)}$, Roberto Acosta ${ }^{(2)}$, and James Nessel ${ }^{(2)}$ \\ (1) Analex Corporation, Cleveland, $\mathrm{OH}, 44135$, www.analex.com \\ (2) The NASA Glenn Research Center, Cleveland, OH, 44135
}

\begin{abstract}
Introduction
The Tracking and Data Relay Satellite System (TDRSS) is a constellation of geosynchronous satellites, which are the primary source of space-to-ground voice, data and telemetry for the Space Shuttle [1]. The satellites also provide communications with the International Space Station and scientific spacecraft in low-Earth orbit such as the Hubble Space Telescope. Integral to the design of the TDRSS class of satellites is an architecture consisting of a multiple access (MA), S-band, phased array antenna. This MA system receives and relays data simultaneously from five lower data-rate users and transmits commands to a single user.

The TDRSS-Continuation Project is the follow-on to the TDRSS class of satellites identified as F1-F10. The specific goals are to enhance the MA array antenna elements for greater on-axis gain, simultaneous polarization capability, and increased beam width. The design specifications for the enhanced MA antenna elements are summarized in Table 1. This paper describes a study (design, fabrication, and testing) of several modified antenna elements for the enhanced MA phased array. Several antenna elements were investigated including a helix antenna, a novel short backfire antenna excited with a circular waveguide, and a corrugated horn antenna. The results for each element in terms of performance; fabrication, assembly and tuning complexity; size and mass are compared below.
\end{abstract}

Table 1 TDRSS Enhanced MA antenna element specifications.

\begin{tabular}{|l|l|}
\hline Frequency $(\mathrm{GHz})$ & $2.2-2.3$ \\
\hline Directivity $(\mathrm{dBi})$ & 15 \\
\hline Axial Ratio $(\mathrm{dB})$ & $>-5 \mathrm{~dB}$ \\
\hline Polarization & Simultaneous LHCP and RHCP \\
\hline Beam width (degs) & $+/-20$ \\
\hline Return loss $(\mathrm{dB})$ & $\leq-20$ \\
\hline Isolation $(\mathrm{dB})$ & $\leq-15$ \\
\hline
\end{tabular}

\section{Helix Antenna}

The program first fabricated and tested a helix antenna using the design for the current TDRSS F1 MA transmit phased array element [2]. The helix element consists of a thin copper wire wound into a helical pattern (See Figure 1). The helical wire is supported in the center by an electromagnetically transparent post, and the base is surrounded by a cup to isolate the element from its surrounding helix elements in the array. A coaxial transformer was designed to match the helix to $50 \Omega$ coaxial cable. Simulations for the helix, as well as the other antennas described here, used the three-dimensional electromagnetic software Microwave Studio [3]. Since the design was based on the 
element for the TDRSS F1 MA, the design frequency was slightly lower than that specified in Table 1.

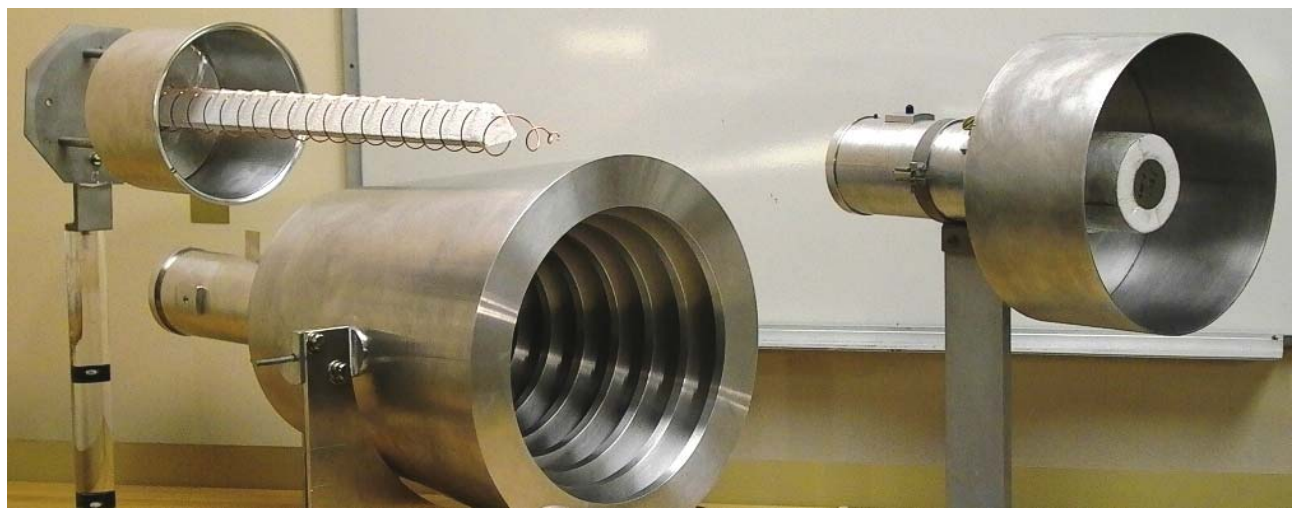

Figure 1 (From left) Helix, corrugated horn and circular waveguide excited short backfire antennas.

Fabrication of the helix was fairly straightforward. Assembling the helical wire on the center post while maintaining the helix diameter and spacing proved to be the most challenging aspect. The return loss was extremely sensitive to the spacing between the helix wire and the ground plane of the cup, as well as the cup base geometry. For example, early fabrication attempts used a 0.060 " thick triangular riser plate to support the helix, which was placed on the cup bottom. This resulted in return loss values completely out of specification. As shown in Figure 2a, removing the triangular plate resulted in excellent return loss values. Figure $2 b$ shows good agreement between the measured and simulated co-polarization, and cross-polarization.

The helix naturally provides circular polarization. However, achieving dual polarization will require placing two helix antennas with opposite helical windings side by side, or a dual feeding arrangement.

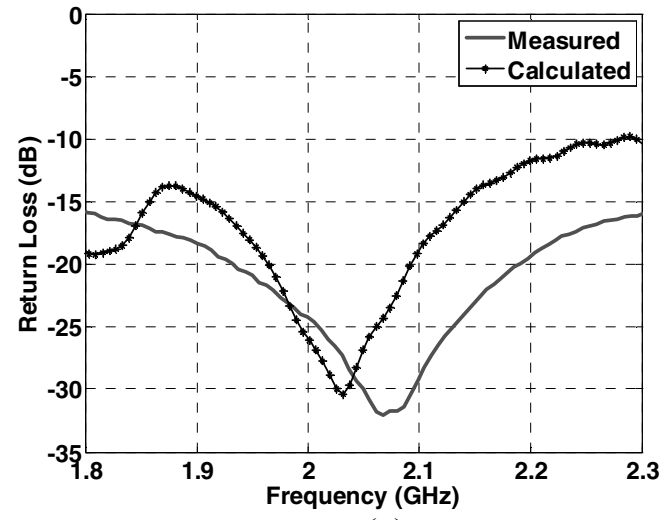

(a)

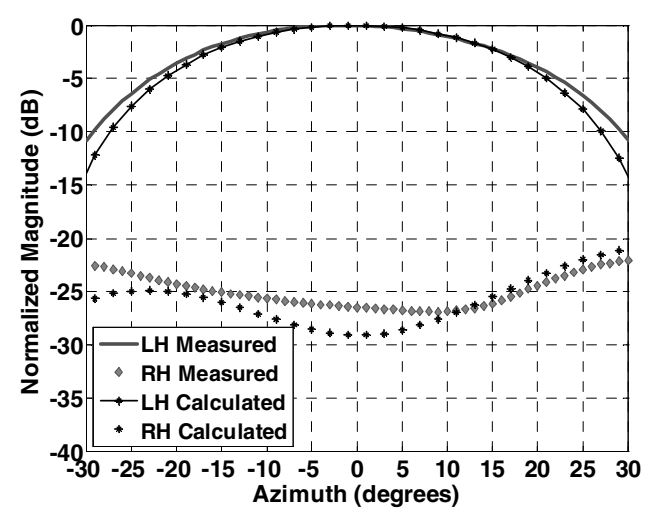

(b)

Figure 2 Measured and simulated (a) return loss, and (b) co-polarization, and crosspolarization for helix antenna.

\section{Circular Waveguide Excited Short Backfire Antenna}

The second antenna is a short backfire antenna (SBA). The SBA is a dual reflector system consisting of a circular waveguide feed, circular disk subreflector, and a circular 
cup. This antenna is shown on the right hand side of Figure 1. To achieve circular polarization, a compact, 6-post polarizer is integrated into the circular waveguide similar to that described in [4]. The circular waveguide also includes an orthomode transducer (OMT) with coaxial ports to achieve dual polarization. This reduces the overall length of the OMT and polarizer to about 11 " compared to about 32 " for a commercially available model.

Compared to the helix, design and fabrication were more complex since the polarizer and OMT required several additional components. Assembly was fairly straightforward, but the antenna required fine tuning, which was complicated by the additional variables of the polarizer screw depths, coaxial port pin lengths, and the subreflector height above the circular waveguide. Figure 3 shows the measured and simulated return loss for port 2 (port 1 results were similar) and the isolation for both ports. The isolation is not within the specification of $<-15 \mathrm{~dB}$. The presence of the subreflector makes this challenging since it introduces additional reflected energy, which causes degradation of the isolation. Measured farfield patterns will be presented, which show very good agreement with simulation, and the specifications were met for directivity and axial ratio.

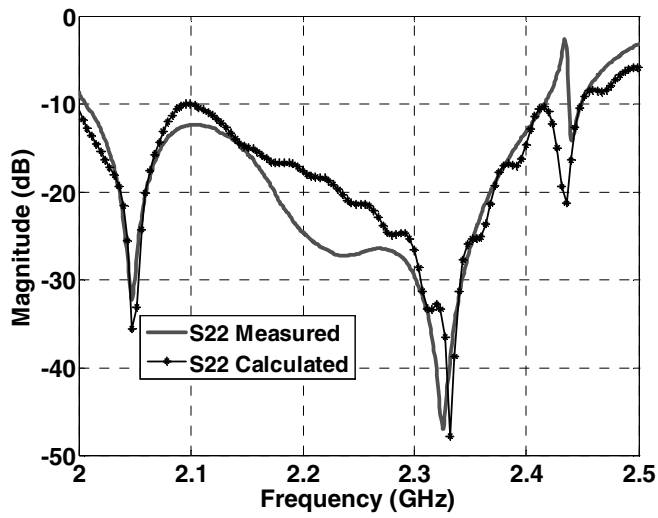

(a)

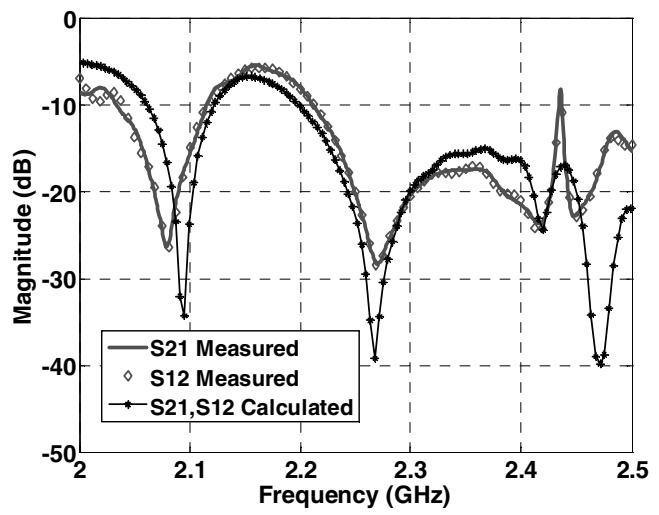

(b)

Figure 3 Measured and simulated (a) return loss, and (b) isolation for short backfire antenna.

\section{Corrugated Horn Antenna}

Lastly, a corrugated horn antenna was designed with the use of a moment of methods code for rotationally symmetric feeds [5]. The horn is fed using a similar OMT and polarizer as described above for the SBA. The fabrication complexity of this element increased over the other two because of the horn corrugations which had to be machined. However, assembly was straightforward requiring only a flange connection between the horn and the OMT/polarizer. Tuning was also straightforward requiring only minor adjustments to the polarizer screws and the coaxial pins. Figure 4 shows the return loss and isolation for port 1 (port 2 results are similar). The results easily meet the specifications for both. Measured farfield patterns will be presented, which show very good agreement with simulation, and the specifications were met for directivity and axial ratio. The disadvantages of the corrugated horn are size and mass as summarized in Table 2. However, no attempt was made to minimize the size and mass for the prototype described here. Electroforming and filling the waveguide with dielectric hold potential for significant size and mass reduction. 


\section{Summary}

We compare three antennas, which are candidates for the TDRSS-Continuation enhanced MA array antenna elements. Measured and simulated data show very good agreement for all antenna elements. All of the antennas meet the specifications with the exception of the SBA isolation. However, improvements can likely be made with further design efforts.

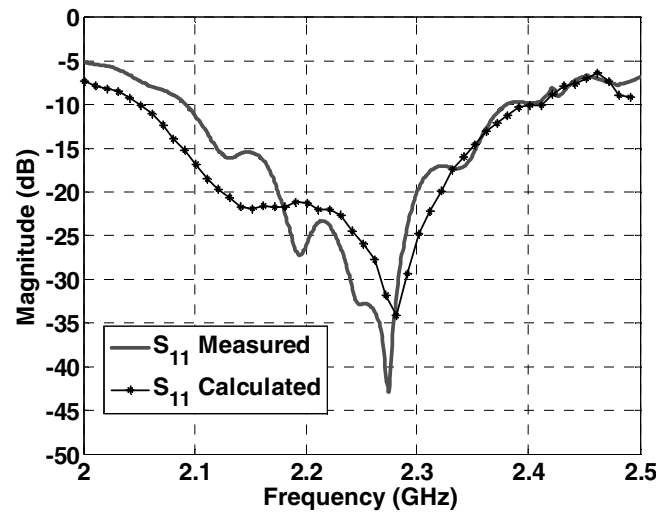

(a)

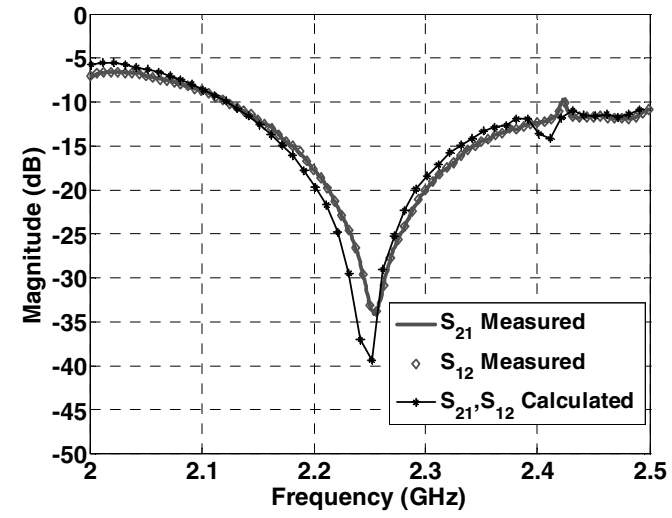

(b)

Figure 4 Measured and simulated (a) return loss, and (b) isolation for corrugated horn antenna.

Table 2 Summary of size and mass for the helix, short backfire and corrugated horn antennas.

\begin{tabular}{|l|l|l|l|l|l|}
\hline & $\begin{array}{l}\text { Mass } \\
\text { (pounds) }\end{array}$ & $\begin{array}{l}\text { Size } \\
\text { Length x } \\
\text { diameter } \\
\text { (inches) }\end{array}$ & $\begin{array}{l}\text { Fabrication } \\
\text { Complexity }\end{array}$ & $\begin{array}{l}\text { Assembly } \\
\text { Complexity }\end{array}$ & $\begin{array}{l}\text { Tuning } \\
\text { Complexity }\end{array}$ \\
\hline Helix & 0.66 & $23 \times 6$ & Low & Medium & Medium \\
\hline SBA & 6.0 & $15.2 \times 10.6$ & Medium & Medium & High \\
\hline Horn & 77.8 & $28.5 \times 15$ & Medium & Low & Low \\
\hline
\end{tabular}

\section{References:}

[1] Web Site for Goddard Space Flight Center TDRSS Project: http://tdrs.gsfc.nasa.gov/Tdrsproject/.

[2] G. Wong and T. Tamakawa, TDRSS Multiple Access Transmit Phased Array, TRW Report No. 2900-330-013.

[3] www.cst.com

[4] B. Subbarao and V. F. Fusco, Compact Coaxial-Fed CP Polarizer, IEEE Antennas and Wireless Propagation Letters, Vol. 3, 2004, pps. 145-147.

[5] Paynter, G.F., Teh-Hong Lee, Burnside, W.D., "Expansion of existing EM workbench for multiple computational electromagnetics codes," IEEE Antennas and Propagation Magazine, Vol 45, No.3, June 2003, pp. 122-127. 